mercial district, they cannot be said to be derived from, or rendered applicable to, one class of mechanics only. A greater tendency also appeared to me to exist, to the generation of organic disease, perhaps especially that of the liver, in individuals of highly bilious nervous temperament; and this bypothesis would appear to be partly confirmed by the observation of a recent writer, who mentions the comparative frequency of carcinoma of the uterus (in a number of cases treated by him) in females of highly bilious temperament.

With regard to the utility of a knowledge of the temperaments in surgical diseases and accidents, the principal benefit that I conceive to be derived is, in many instances, to render the diagnosis and prognosis more clear and certain, and thus afford material assistance to him in some cases.

It has been my endeavor, in the foregoing remarks, to treat as concisely as possible some of the leading points connected with a subject that admits of very extensive research, even if one not capable of affording material assistance to the physician in the diagnosis and treatment of disease, at least one that might tend to elucidate some of those as yet hidden phenomena which we meet with in practice; and my object, in giving publication to these brief and imperfect remarks is-through the medium of the Lancet -if possible, to draw attention to the subject. It is now a year since most of the foregoing notes were made, at a time when enjoying considerable opportunities for so doing, and my subsequent experience has tended to confirm the opinions I entertained then.-London Lancet.

\title{
CASE OF INVERSION OF THE UTERUS.
}

By घ. Fisher, M.D., of Waynesville, Ohio.

$\mathrm{ON}_{\mathrm{N}}$ the $26 \mathrm{th}$ of September, 1835, at 10 o'clock, A. M., I was called to Mis. D-, in labor. She was about 35 years of age, and had given birth to several children-said that two weeks previously, in attending the funeral of a relative, she rode several uniles in a farm-waggon over rough ronds, which excited pain in the loins and hips, and weakness of the inferior extremities, attended with difficulty of locomotion; all of which continued up to the morning of the $26 \mathrm{th}$.

From the day of the funeral she had felt no motion of the fotus, and, to use her own language, she was " eight months gone in pregnancy." The pains were slight, irregular and transient. Upon examination I found the pelvis unusually large, the os uteri well dilated, and the membranes protruding. The temperature of the skin was natural, pulse regular, and bowels open.

As I conceived there was but little to fear, I deemed it prudent to give nature time to effect her purpose. Ten grains of pulris Doveri were administered, which procured an hour's repose. The pains then returned, but continued feeble an hour longer without any appreciable change, when suddenly a violent throe thrust foetus, placenta, and body of the uterus beyond the labia exteria. 
The fotus was very small and putrid; the funis umbilicalis, as nearly as I could estimate it (not having any means of measurement), was eight inches in length. The placenta was detached, and complete inversion of the uterus had taken place. I was shocked for a moment with the condition of my patient, but knowing there was no time to be lost, I immediately cominenced an attempt to return the uterus. I passed the index of the right hand into the vagina, then carried it round the tumor, till I became fully satisfied as to the condition of the parts. The os uteri lonked into the pelvic cavity, and the finger could not be brought into contact with it.

The uterus was as flaccid as a wet bladder; a circurnstance which inspired a ray of hope that something might be done to relieve the patient from a situation but little more desirable than death. I placed the fingers of my right hand against the fundus of the uterus, pressing it gently upwards in the direction of the axis of the inferior strait, while the left hand. was placed over the hypogastric region to prevent the uterus from rising into the abdomen. I carried my right hand up the vagina a sufficient extent to enable me to return the uterus, which was done with less diffculty than could have been anticipated. Not the slightest contraction of either the fundus, body, or neck of the organ, took place during the operation. I made an attempt to withdraw my hand, and the fundus followed it. And notwithstanding frictions were made over the hypogastrium with the left hand, while the knuckles of the right were caused to press against the fundus of the organ within, the uterus still remained flaccid, and during some minutes manifested no disposition to contract. I ordered one drachm of secale cornutum infused in six ounces of water, two ounces of which were to be given every ten minutes; the third portion produced contractions, and by grasping the fundus between the thumb and fingers of the left hand, through the walls of the abdomen, in less than fifieen minutes the contractions became so violent that my hand was forced out into the vagina. No further difficulty ensued. The hemorrhage was less than in an ordinary case of labor at the full period of utero-gestation, and neither pain nor syncope occurred prior to or during the inversion of the organ. The practicability of returning the uterus when a complete inversion has taken place, has been doubted by Dr. Dewees, he not having been able to succeed in those cases that came under his observation, on account of the contracted condition of the cervix uteri, and while the fundus and body remained in a relaxed condition. He conceived that in such cases the disproportion between the several parts of the organ was so great, and its attachment with the pelvis so low, that any attempt at restoration must prove abortive. In the case of Mrs. D. an injury had been sustained by travelling in a farm uraggon over rough roads-a dead foetus retained in the uterus some days-ihe funis umbilicalis some eight inches in length, and when the labor came on, the fotus appeared to be expelled almost entirely by the action of the abdominal nuscles. In Dewees's cases, atony of the fundus and body existed, while the cervix and os uteri retained their contractile power. In the case under consideration the fundus, body, cervix and os uteri were in a state of extreme atony.-Illinois Medical and Surgical Journal. 